\title{
Toxoplasma gondii Hsp20 is a stripe-arranged chaperone-like protein associated with the outer leaflet of the inner membrane complex
}

\author{
Natalia de Miguel ${ }^{\star}$, Maryse Lebrun $^{\dagger}$, Aoife Heaslip ${ }^{\ddagger}$, Ke Hu§, Con J. Beckers ${ }^{\|}$, Mariana \\ Matrajt ${ }^{\ddagger}$, Jean F. Dubremetz ${ }^{\dagger}$, and Sergio O. Angel ${ }^{{ }^{\prime}, 1}$ \\ "Laboratorio de Parasitologia Molecular, Instituto de Investigaciones Biotecnológicas-Instituto \\ Tecnológico de Chascomús, Chascomús, Argentina \\ †UMR5235 CNRS, Université de Montpellier 2, Place E Bataillon, 34095 Montpellier, France \\ ‡Department of Microbiology and Molecular Genetics, University of Vermont, Burlington, VT 05405, \\ U.S.A. \\ §Department of Biology, Indiana University, Bloomington, IN 47405, U.S.A. \\ "Department of Cell and Developmental Biology, University of North Carolina at Chapel Hill, Chapel \\ Hill, NC 27599, U.S.A.
}

\begin{abstract}
Background information-Toxoplasma gondii is among the most successful parasites, with nearly half of the human population chronically infected. $T$. gondii has five sHsps [small Hsps (heatshock proteins)] located in different subcellular compartments. Among them, Hsp20 showed to be localized at the periphery of the parasite body. sHsps are widespread, constituting the most poorly conserved family of molecular chaperones. The presence of sHsps in membrane structures is unusual.
\end{abstract}

Results-The localization of Hsp20 was further analysed using high-resolution fluorescent light microscopy as well as electron microscopy, which revealed that Hsp20 is associated with the outer surface of the IMC (inner membrane complex), in a set of discontinuous stripes following the same spiralling trajectories as the subpellicular microtubules. The detergent extraction profile of Hsp20 was similar to that of GAP45 [45 kDa GAP (gliding-associated protein)], a glideosome protein associated with the IMC, but was different from that of IMC1 protein. Although we were unable to detect interacting protein partners of Hsp20 either in normal or stressed tachyzoites, an interaction of Hsp20 with phosphatidylinositol 4-phosphate and phosphatidylinositol 4,5-bisphosphate phospholipids could be observed.

Conclusions-Hsp20 was shown to be associated with a specialized membranous structure of the parasite, the IMC. This discontinuous striped-arrangement is unique in $T$. gondii, indicating that the topology of the outer leaflet of the IMC is not homogeneous.

\section{Keywords}

chaperone; gliding-associated protein; inner membrane complex; small heat-shock protein (sHsp); Toxoplasma gondii; transfection

(C) 2008 Portland Press Ltd

1To whom correspondence should be addressed (sangel@intech.gov.ar).. 


\section{Introduction}

The protozoan Toxoplasma gondii is an obligate intracellular parasite that is able to infect and succeed in most nucleated cells of warm-blooded animals. Recent work on apicomplexa has been focused on the cellular structures and molecules involved in their ability to invade mammalian cells, their peculiar cell cycle, mechanisms of cell division and the presence of several unique organelles. An important structure that received particular attention is the pellicle of the parasite, which consists of the plasma membrane and of the IMC (inner membrane complex). At the cytoplasmic face, the IMC interacts with a set of 22 subpellicular microtubules via an organized array of IMPs (intramembranous particles; Morrissette et al., 1997). Cell division occurs by packaging two daughter cells within a single mother cell during mitosis, in a process termed endodyogeny (van der and Piekarski, 1967). A critical step towards building daughter cells is the initiation of a new IMC, which forms early during parasite division (Hu et al., 2002a). It is structurally associated with a network formed by a group of proteins weakly related to intermediate filaments (Mann and Beckers, 2001; Hu et al., 2002a; Gubbels et al., 2004). It has been hypothesized that the IMC network of the daughters at the early stage of cell division is flexible, allowing for growth, but proteolytic processing of IMC components is then thought to stabilize the structure in the mature cell (Hu et al., 2002a; Mann et al., 2002).

The IMC also plays a critical role as an anchor for the gliding apparatus of the parasite, which is based on an intricate actin-myosin motor system, sandwiched between the parasite's plasma membrane and the IMC (Dobrowolski and Sibley, 1996; Gaskins et al., 2004). A class XIV myosin (Meissner et al., 2002), paired with myosin light chain and rooted to the IMC by two GAPs (gliding-associated proteins), is involved in driving the parasite motility (Gaskins et al., 2004).

All organisms respond to environmental stress by synthesizing Hsps (heat-shock proteins), including the group of sHsps (small Hsps) characterized by a molecular mass ranging between 15 and $42 \mathrm{kDa}$ (Coucheney et al., 2005). sHsps are considered as ATP-independent chaperones that interact with partially folded target proteins to prevent their aggregation and precipitation under conditions of cellular stress (Haslbeck et al., 2005). sHsps are present in a large variety of cellular compartments, including the cytoplasm, nucleus, chloroplast, mitochondria and endoplasmic reticulum in higher plant cells (Nakamoto and Vigh, 2007). In addition, multiple members of this family could be present in the same cellular compartment, indicating a possible function redundancy (Nakamoto and Vigh, 2007). Our preliminary work (de Miguel et al., 2005) described the identification and characterization of the T. gondii $\alpha$-crystallin/sHsp family. Surprisingly, although most unicellular organisms have just one or a few cytosollocated sHsps, $T$. gondii has five different sHsps located in three different cellular compartments (the cytoplasm, mitochondria and parasite pellicle), suggesting that targets and functions might be different (de Miguel et al., 2005). It is known that some molecular chaperones facilitate formation of the cytoskeleton elements, influence their function and protect their constituent proteins against misfolding (Gusev et al., 2002). Recently, potential links between sHsps and membrane functions have attracted increasing interest (Nakamoto and Vigh, 2007). For example, the $\alpha$-crystallin chaperone, a major component of lens in vertebrate eyes, has been shown to bind the plasma membrane and synthetic phospholipids vesicles stabilizing heat-stressed membranes by increasing membrane physical order (Cobb and Petrash, 2000; Torok et al., 2001; Coucheney et al., 2005).

Despite the central role of membrane and cytoskeleton assembly in providing mechanical strength, cell development, maintenance of cell shape and structural integrity, little is known about the molecular composition and function of the pellicle in T. gondii parasites. Based on our previous observation of the peripheral localization of $T$. gondii Hsp20 along the parasite 
body (de Miguel et al., 2005), we performed an in-depth analysis of the location and the topology of $\mathrm{Hsp} 20$ as well as determined its interaction partners to explore the potential role of this protein in the membrane of the parasite.

\section{Results}

\section{Hsp20 is a stripe-arranged chaperone homologue associated with the IMC}

We have previously shown that mouse anti-Hsp20 serum showed a crescent-shaped pattern at one end of the parasite, and also in the pellicle (de Miguel et al., 2005). Here, the Hsp20 localization was re-examined by double IFA (immunofluorescent assay) employing a rabbit anti-Hsp20 serum and a monoclonal antibody directed against the micronemal protein MIC2. It is known that micronemal proteins in $T$. gondii produce a discrete signal at the apical end of the parasite when detected by immunofluorescence (Achbarou et al., 1991). Hsp20 labelling could be detected along the parasite body, but it was weaker in the apical compartment where MIC2 localized (Figure 1A). In fact, Hsp20 signal presented a similar pattern as IMC1 and GAP45 (45 kDa GAP; results not shown); other previously described proteins localized at the IMC of the parasite (Hu et al., 2002a; Gaskins et al., 2004). In fact, the Hsp20 signal along the parasite body was weaker in the apical compartment where MIC2 localized (Figure 1A). Hsp20 labelling was also evident in budding cells (Figure 1B, upper panel), indicating its association with the IMC (Hu et al., 2002a). Most interestingly, Hsp20 appeared to be arranged in discontinuous stripes, arranged in paths running through almost the entire parasite body (Figure 1B, lower panel). To corroborate these findings, subcellular localization of Hsp20 was also analysed by transgenic parasites expressing YFP (yellow fluorescent protein)- and Ty-tagged Hsp20. In both cases, Hsp20 localized to the periphery, showing a discontinuous stripearranged pattern along the pellicle of the parasite (Figures 1C and 1D). Figure 1(D) (right panel) also shows Hsp20 labelling at the IMC of daughter cells. Hsp20 could also be found in the conoid region in some transgenic parasites (results not shown), which is similar to what has previously been observed (de Miguel et al., 2005).

\section{Hsp20 stripes follow the same trajectory as the subpellicular microtubules}

In order to analyse the relationship between the stripe arrangement of Hsp20 and subpellicular microtubules, parasites were co-transfected with Hsp20-YFP and mCherry- $\alpha$-tubulin1 constructions. Hsp20 appeared to be arranged in stripes, following the same trajectories of the subpellicular microtubules at the periphery of the parasite (Figure 1E). However, the Hsp20 stripes were discontinuous and much longer than the length of the subpellicular microtubules.

\section{Hsp20 is localized to daughter cells in later stages of parasite replication}

In order to understand how Hsp20 is recruited to the IMC, Hsp20 localization was examined during the endodyogeny process by IFA. We performed co-localization studies of Hsp20 and IMC1 at different stages of cell division. As can be seen in Figure 2, the network of the new daughter cells can be detected from a very early stage of endodyogeny (IMC1 panel) as described by Mann et al. (2002). However, the Hsp20 labelling of the daughters cells only became apparent at later stages of daughter cell construction (Figure 2). In addition, and in contrast with what was observed for IMC1-YFP, in later stages Hsp20 labelling was also visible in the mother IMC (Figure 2).

\section{Hsp20 is localized in the outer leaflet of the IMC}

To determine the Hsp20 localization at higher resolution, we carried out immunoelectron microscopy using anti-Hsp20 antibody in both intracellular and extracellular parasites. The Hsp20 was detected at the IMC of the parasite (results not shown). Moreover, the IMC localization of Hsp20 was particularly clear in pellicle preparations (Gaskins et al., 2004), 
where the plasma membrane is separated in many places from the underlying IMC. Using this approach, we discovered that the Hsp20 antibody reacted specifically with the IMC, but not with the plasma membrane (Figure 3 ). Furthermore, the gold labelling was clearly found only on the side of the IMC facing the parasite plasma membrane (Figure 3).

\section{Detergent extraction profile showed that Hsp20 is a membrane-associated protein}

To examine the Hsp20 pellicle association, the extractability of this protein in detergents was also evaluated. We tested Hsp20 solubility in TBS (Tris-buffered saline), observing that Hsp20 remained as an insoluble protein after parasite disruption (Figure 4A). To analyse Hsp20 detergent extraction profile, preparations were subjected to a sequential extraction process with Triton X-100 and sodium DOC (deoxycholate). As can be seen in Figure 4(A), Hsp20 was present in the IMC-enriched fraction. The extraction profile observed for Hsp20 showed a behaviour indistinguishable from that of GAP45, a previously described glideosome membrane protein associated with the IMC facing the parasite plasma membrane (Gaskins et al., 2004), indicating that Hsp20 is associated with the membrane of the IMC. In contrast, the membrane skeleton protein IMC1 remained insoluble under either conditions as previously described (Johnson et al., 2007). Given that actin is a protein found both in a soluble form (globular monomer form) and in the cytoskeleton network (Dobrowolski et al., 1997; Patron et al., 2005), it was used as a control in this set of experiments.

\section{Hsp20 interacts with phospholipids in vitro}

In order to identify putative biomolecules interacting with Hsp20, different sets of experiments were designed. As a first step, $\left[{ }^{35} \mathrm{~S}\right]$ methionine $/\left[{ }^{35} \mathrm{~S}\right]$ cysteinemetabolically labelled parasites were subjected to IP (immunoprecipitation) assays. When cells were lysed in a TX-100 (Triton $\mathrm{X}-100)$-containing buffer, an approx. $20 \mathrm{kDa}$ protein was immunoprecipitated with a rabbit anti-Hsp20 antibody (Figure 4B). This protein was confirmed to be Hsp20 by a MALDI-TOF (matrix-assisted laser-desorption ionization-time-of-flight) analysis. A number of faint bands were also observed in the immunoprecipitate (Figure 4B). To date, we have not been able to identify these proteins due to the lack of sufficient material. To eliminate the possibility that the detergent used in the lysis buffer could disrupt Hsp20 complexes, IPs with different detergents and at different concentrations were performed. In addition, since Hsp20 is a chaperone protein induced by heat stress conditions, metabolically labelled parasites were incubated for $2 \mathrm{~h}$ at $42^{\circ} \mathrm{C}$ before IP analysis with anti-Hsp20. Nevertheless, the faint bands observed with TX-100 IP buffer were not present in most of the assays performed, indicating that Hsp20 probably does not exist in a stable protein complex (results not shown).

Prompted by our observation of its association with IMC membrane, interaction of Hsp20 with lipids was analysed by a dot-blot overlay assay. In this qualitative assay, nitrocellulose filters on which 15 different lipids have been immobilized were probed with rHsp20 (recombinant Hsp20)-His-tag fusion protein. Figure 4(C) shows that rHsp20 interacts with phosphatidylinositol 4-phosphate or phosphatidylinositol 4,5-bisphosphate, suggesting that Hsp20 is a phospholipid-binding protein. By contrast, neither control (where rHsp20 was omitted) nor $\mathrm{His}_{6}$-tagged rHsp28, an sHsp located in parasite mitochondria (de Miguel et al., 2005), interacted with any of these lipids (Figure 4C and results not shown).

\section{Discussion}

In the present study, we observed that Toxoplasma Hsp20 is associated with the membrane of the IMC. Since only a few sHsps are described to be associated with membranes in nature (see Nakamoto and Vigh, 2007), we consider such a finding to be very remarkable. Interestingly, the IMC is a specialized compartment forming the pellicle of apicomplexan parasites. In $T$. gondii, the pellicle is composed of three layers of membranes. The plasma membrane is closely 
apposed to the IMC, which is composed of flattened vesicles joined by sutures in a patchwork fashion (Vivier and Petitprez, 1969; Porchet and Torpier, 1977). The subpellicular microtubules are likely associated with the IMC via IMP particles, both organized into a gentle spiral along the parasite body. The Toxoplasma Hsp20 protein, a homologue of the chaperone of sHsp/ $\alpha$-crystallin family, is localized to the outer face of the IMC, similar to glideosome proteins (Gaskins et al., 2004). A detailed analysis showed that Hsp20 appeared as discontinuous stripes down the body of the parasite. It is interesting to point out that this pattern is not shared by other IMC/cytoskeletal proteins of the parasite, like GAP45, IMC1 and microtubules. Recently, Gilk et al. (2006) described a similar pattern for PhIL1

[photosensitized INA (iodonaphthalene-1-azide)-labelled protein 1], but in this case, the stripes were apparent only in DOC-extracted parasites. The observation that Hsp20 is located at the IMC and Hsp20-stripes follow the same trajectories as those of the subpellicular microtubules, but extend further back to the posterior end of the parasite is more consistent with the possibility that Hsp20 follow double-row IMPs that could be underlying the microtubules (Porchet and Torpier, 1977; Morrissette et al., 1997).

An approach to address the biological role of Hsp20 could be to identify interacting biomolecules. So far, we have not been able to identify any binding partners for Hsp20. Since Hsp20 belongs to the sHsp family, it could be inferred that (i) the complexes formed are unstable and/or dynamics; (ii) only a fraction of the Hsp20 population would be interacting with proteins of the parasite; (iii) Hsp20 could be interacting with other proteins only under specific situations (e.g. during parasite gliding, stress etc.) or (iv) there is no protein interacting with Hsp20. Based on these possibilities, future studies to identify transient Hsp20 partners should be performed using different strategies like IP assays under cross-linking conditions.

It is worth noting that $T$. gondii Hsp20 exhibited binding to phosphatidylinositol 4-phosphate and phosphatidylinositol 4,5-bisphosphate, a minor class of lipids of the parasite (Foussard et al., 1991; Gupta et al., 2005; Coppens, 2006). PPIs (phosphatidylinositol phosphates) serve as important regulators in cellular membrane dynamics and are concentrated at the specific subcellular locations acting as compartment-specific recognition signals for cytosolic binding partners and assist in the targeting of peripheral membrane proteins to these membranes (De Matteis and Godi, 2004a, b; Roth, 2004;Yamashita et al., 2006). These phosphoinositides also contribute to membrane dynamics by recruiting specific factors such as adaptor proteins, lipid transfer proteins, and components of the endosomal sorting complex required for transport (Hanada et al., 2003; Raiborg et al., 2003), being essential regulators of many cell functions, including signalling through hormone and growth factor receptors, cell survival versus apoptosis, motility and membrane trafficking (Michell et al., 2006). Further studies should be performed to throw light on the binding properties of these lipids with Hsp20 and its role.

A potential link between $\mathrm{sHsp}$ and membrane function has recently emerged over the past years where sHsps strongly modulate the membrane properties of anionic phospholipids, suggesting a protection by sHsps at both the thermal extremes (Tsvetkova et al., 2002). For example, a critical role of sHsps in controlling the physical state, bilayer stability and thereby integrity of membranes via specific lipid interactions has been established with Hsp17 from Synechocystis PCC 6803 (Torok et al., 2001). Based on our results, it could be hypothesized that Hsp20 could possess a similar role in the IMC of Toxoplasma. The apparent late recruitment of Hsp20 to the daughter IMC during parasite replication suggests that this protein might be involved in the maturation of the newly assembled IMC. At the last stage of this process, when the IMC and underlying network of the mother cell are destroyed, the network in the daughter parasites would need to switch rapidly from a loose to a rigid conformation in order to lend structural support to the newly generated parasites (Mann et al., 2002). The labelling of Hsp20 in the last stages of the endodyogeny process could suggest a possible role 
in the switch of structural properties of the network that contribute to fluidity modification of mature IMC.

Our study clearly shows that Hsp20 localizes to the outer surface of the IMC of the parasite in a stripe-like organization, possibly via interaction with PPIs. Taken together the results indicate that the topology of IMC surface is not homogeneous. It is clear that further investigations are needed to identify the role of Hsp20 in the biology of $T$. gondii. In spite of this, taken together the results presented in this paper and the growing evidence of the involvement of sHsps in other cellular functions beyond their role in thermo-tolerance and chaperone properties (Garrido et al., 2001; Parcellier et al., 2003; Arrigo, 2005), we could infer that Hsp20 biological function could be related to protect and/or modulate membrane properties of the IMC, via assisting proteins folding under certain conditions, protecting the membrane IMC under stress and/or modulating membrane fluidity. How Hsp20 affects the physiology of $T$. gondii cells (e.g. replication and/or gliding) is still unclear and captivating. In addition, the unique stripe pattern of Hsp20 is also highly intriguing and an exciting subject for future studies. Another interesting question is how the Hsp20 is targeted to the IMC, giving this characteristic pattern. In this regard, we are currently carrying out experiments to elucidate the biological role of Hsp20 and how its arrangement is regulated.

\section{Materials and methods}

\section{Culture of parasites}

T. gondii was maintained by serial passage in confluent cultures of HFFs (human foreskin fibroblasts) cells in $\alpha$-minimal essential medium (Mediatech, Herndon, VA, U.S.A.) supplemented with 10\% (v/v) fetal bovine serum (Hyclone, Logan, UT, U.S.A.), $2 \mathrm{mM}$ glutamine, 100 i.u. (international unit)/ml penicillin and $100 \mu \mathrm{g} / \mathrm{ml}$ streptomycin. Parasites were harvested by syringe passage followed by centrifugation at $1000 \mathrm{~g}$ for $8 \mathrm{~min}$.

\section{Detergent extraction}

A pellicle fraction was prepared following the protocol described by Johnson et al. (2007). Tachyzoites $\left(10^{8}\right.$ parasites) were extracted in $1 \%$ TX-100 in PBS $(154 \mathrm{mM} \mathrm{NaCl}, 1.54 \mathrm{mM}$ $\mathrm{KH}_{2} \mathrm{PO}_{4}$ and $2.71 \mathrm{mM} \mathrm{Na}_{2} \mathrm{HPO}_{4}, \mathrm{pH}$ 7.4) and $1 \mathrm{mM}$ PMSF for $30 \mathrm{~min}$ on ice. TX-100insoluble material was collected by centrifugation at $10000 \mathrm{~g}$ for $10 \mathrm{~min}$, washed once in $1 \%$ TX-100 in PBS, and extracted for $30 \mathrm{~min}$ on ice in $1 \% \mathrm{TX}-100$ in TBS $(100 \mathrm{mM}$ Tris/ $\mathrm{HCl}$ and $0.9 \% \mathrm{NaCl}, \mathrm{pH}$ 7.5). The TX-100/TBS-soluble and -insoluble material was collected by centrifugation at $10000 \mathrm{~g}$ for $10 \mathrm{~min}$ at $4^{\circ} \mathrm{C}$. The TX-100/TBS-insoluble material was subsequently extracted for $30 \mathrm{~min}$ on ice in $1 \%$ DOC in TBS and PMSF. DOC-soluble material was separated from insoluble material by centrifugation as it was described above (Mann and Beckers, 2001; Gaskins et al., 2004). Samples were run on SDS/12\% PAGE gels and transferred to nitrocellulose. Blots were probed with the following antibodies: rabbit antiHsp20 (1:1000), rabbit anti-IMC1 (1:2000); monoclonal anti-SAG1 (1:500), rabbit antiGAP45 (1:2000), monoclonal anti-Actin (1:500) followed by either alkaline phosphataseconjugated anti-rabbit or anti-mouse secondary antibodies (1:30 000) (Sigma-Aldrich).

\section{Plasmid construction and expression in Toxoplasma}

To generate the Hsp20-Ty and Hsp20-YFP constructs, the ORF (open reading frame) was amplified using the primers 5'-AGATCTATGAGTTGCTGTGGCGGTAC-3' and 5'CCTAGGTGAATTTTTTGAGGCCTTC-3'. BgIII and AvrII restriction sites were engineered into the 5'- and 3'-primers respectively. The resulting PCR product of the expected size was excised using a Qiaex II Gel Extraction kit (Qiagen), cloned in pGEM-T vector (Promega), and sequenced employing the ABI PRISM Big Dye Terminator Cycle Sequencing Ready Reaction kit (PerkinElmer Applied Biosystems). The cloned ORFs were digested with BgIII 
and AvrII and inserted between the BgIII and AvrII sites in the plasmids ptub-MIC3-Ty (Cerede et al., 2005) and ptubIMC1-YFP/sagCAT (Hu et al., 2002a). Both the plasmids were designed to express the Hsp20 protein with a C-terminal Ty or YFP tag in RH [HXGPRT (hypoxanthinexanthine-guanine phosphoribosyl transferase)-] strain.

Hsp20-Ty and Hsp20-YFP constructs were transfected into Toxoplasma by electroporation and Hsp20-Ty stable transfectants were obtained by selection with $25 \mu \mathrm{g} / \mathrm{ml}$ mycophenolic acid and $50 \mu \mathrm{g} / \mathrm{ml}$ xanthine for three passages before cloning by limiting dilution under drug selection (HXGPRT selection) (Cerede et al., 2005). After expanding the clones, parasites expressing Hsp20-Ty were identified by IFA with anti-Ty antibody (Bastin et al., 1996).

\section{Electron microscopy}

Intracellular and extracellular tachyzoites were resuspended in PBS, pelleted and fixed for 15 min at room temperature $\left(25^{\circ} \mathrm{C}\right)$ with $2 \%(\mathrm{w} / \mathrm{v})$ paraformaldehyde and $0.1 \%$ glutaraldehyde in $0.2 \mathrm{M}$ sodium phosphate buffer ( $\mathrm{pH}$ 7.4). The pellet was dehydrated in ethanol at $-20^{\circ} \mathrm{C}$ and embedded in LRWhite (London Resin Co., Berkshire, U.K.). Thin sections were collected on carbon-coated grids and saturated for $30 \mathrm{~min}$ with $2.5 \%(\mathrm{w} / \mathrm{v})$ skimmed dried milk powder and $0.1 \%$ Tween 20 in PBS (PBS-MT). The grids were floated successively for $1 \mathrm{~h}$ each on rabbit anti-Hsp20 serum diluted 1:50, followed by Protein A-10-nm gold diluted in PBS-MT, with PBS washes between each step. The grids were then stained with uranyl acetate and lead citrate and observed with a Hitachi H700 electron microscope.

A pellicle fraction was prepared from $4 \times 10^{8}$ parasites by sonication and differential centrifugation (10000 $\boldsymbol{g}$ for $10 \mathrm{~min}$ ) (Mann and Beckers, 2001). For pre-embedding immunoelectron microscopy, isolated pellicles were incubated with primary antibody (antiHsp20 at 1:500) diluted in 3\% (w/v) BSA in PBS for $3 \mathrm{~h}$ on ice. The sample was washed in PBS by sedimentation (10000 $g$ for $10 \mathrm{~min}$ ) and resuspension. Incubation with 10-nm goldconjugated Protein A (from Department of Cell Biology, Utrecht University) diluted 1:25 in 3\% BSA in PBS was performed overnight at room temperature. The sample was washed in PBS as described above and fixed in 1\% glutaraldehyde in PBS for 20 min. After another PBS wash, the sample was fixed in 1\% osmium tetroxide in PBS for $20 \mathrm{~min}$, washed with PBS, dehydrated in increasing concentrations of ethanol, embedded in Spurr's resin (Electron Microscopy Sciences) and polymerized overnight at $70^{\circ} \mathrm{C}$. Thin sections were prepared, stained with uranyl acetate and lead citrate, and observed with a Zeiss EM10 electron microscope.

\section{Immunolocalization experiments}

Intracellular parasites were grown for $24 \mathrm{~h}$ in HFFs on glass slides. All further incubations were carried out at room temperature. Cells were washed in PBS and fixed in $4 \%$ formaldehyde for $20 \mathrm{~min}$. After three washes, cells were permeabilized with $0.2 \%$ TX-100 in PBS for 10 min, blocked with 10\% PFBS (fetal bovine serum in PBS) for $20 \mathrm{~min}$, incubated with primary antibody diluted in 2\% PFBS, washed and then incubated with a 1:4000 dilution of Alexa Fluor $^{\circledR}$-conjugated secondary antibody (Molecular Probes). The coverslips were mounted on to microscope slips using Immunomount (Calbiochem). All observations were performed on a Leica DMRA2 microscope or a Delta Vision Wide-field deconvolution system based on an Olympus IX-70 inverted microscope (Applied Precision). Adobe Photoshop (Adobe Systems) was used for image processing.

\section{Hsp20-YFP and mCherry- $\alpha$-tubulin1 co-transfection experiments}

The YFP- $\alpha$-TUBA1 (Hu et al., 2002b) and the mCherryFP- $\alpha$-TUBA1 plasmids were used. One-third of the RH parasites from a T12.5 flask culture $\left(\sim 1 \times 10^{7}\right)$ were transfected by electroporation with $25 \mu \mathrm{g}$ each of ptubHsp20-YFP and ptub-mCherryFP- $\alpha$-tubulin1 plasmid 
DNA, allowed to infect a fresh monolayer of host cells for approx. $20 \mathrm{~h}$, fixed with $4 \%$ formaldehyde for $15 \mathrm{~min}$ at room temperature and observed with a Delta Vision Wide-field deconvolution microscope, using a $\times 100$ NA 1.35 oil objective. Three-dimensional image stacks were collected at $z$-increments of $0.3 \mu \mathrm{m}$, deconvolved images were computed using the point-spread functions and software supplied by the manufacturer, and maximum intensity projections of deconvolved three-dimensional stacks were used as figures.

\section{Metabolic labelling of $T$. gondii}

Parasites were allowed to invade a monolayer of HFF cells. After $14-16 \mathrm{~h}$, cells were incubated in methionine/cysteine-free medium (Mediatech) for $30 \mathrm{~min}$ before the addition of $50 \mu \mathrm{Ci} / \mathrm{ml}$ $\left[{ }^{35}\right.$ S $]$ methionine/[ $\left.{ }^{35} \mathrm{~S}\right]$ cysteine (700 Ci/mM; MP Biomedicals, http://www.mpbio.com).

Parasites were harvested on ice after a $20-24-\mathrm{h}$ incubation at $37^{\circ} \mathrm{C}$ and the IP experiment was performed.

To perform the IP under heat stress conditions, HFF cells grown in two different flasks were infected and $0.25 \mathrm{mCi}\left[{ }^{35} \mathrm{~S}\right]$ methionine/ $\left[{ }^{35} \mathrm{~S}\right]$ cysteine was added after the starvation in methionine/cysteine-free medium. One flask was incubated at $37^{\circ} \mathrm{C}$ for $2 \mathrm{~h}$ (normal condition) and the other was incubated at $42^{\circ} \mathrm{C}$ for $2 \mathrm{~h}$ (stress condition). Parasites were harvested on ice after $2 \mathrm{~h}$.

\section{IP experiment}

Parasites $\left[(5-10) \times 10^{8} \mathrm{ml}^{-1}\right]$ were lysed with IP buffer $(1 \%$ TX-100, $50 \mathrm{mM}$ Tris/HCl, $\mathrm{pH}$ $8.0,150 \mathrm{mM} \mathrm{NaCl}$ and $5 \mathrm{mM}$ EDTA) in the presence of protease inhibitors (P8340; SigmaAldrich) for $4 \mathrm{~h}$ at $4{ }^{\circ} \mathrm{C}$, followed by centrifugation at $16000 \mathrm{~g}$ for $20 \mathrm{~min}$ at $4^{\circ} \mathrm{C}$. Protein ASepharose (Sigma) was pre-incubated at $4^{\circ} \mathrm{C}$ for $4 \mathrm{~h}$ with anti-Hsp20 serum. Anti-Hsp20Protein A-Sepharose was added to the lysis supernatant and the incubation was continued overnight at $4^{\circ} \mathrm{C}$. Immune complexes were washed six times in IP buffer (each wash for 10 $\min$ at $4^{\circ} \mathrm{C}$ ), eluted in SDS/PAGE sample buffer and separated by SDS/PAGE.

Gels containing radiolabelled immune complexes were subjected to fluorography using Enhance (NEN Life Science Products), dried and exposed to a Kodak XOMat AR film.

\section{Dot-blot 'overlay' assays to determine lipid binding}

Membrane arrays (PIPStrips) spotted with 100 pmol of 15 different phospholipids were purchased from Echelon Research Laboratories (Salt Lake City, UT, U.S.A.) and used for protein-lipid overlay assay by following the manufacturer's instructions. Briefly, membranes were blocked with $1 \%$ skimmed dried milk powder in PBST for $1 \mathrm{~h}$ at room temperature. Blocked membranes were incubated with $10 \mu \mathrm{g} / \mathrm{ml} \mathrm{Hsp20-His} \mathrm{or} \mathrm{Hsp28-His-tag} \mathrm{fusion}$ proteins (de Miguel et al., 2005) overnight at $4^{\circ} \mathrm{C}$ with gentle agitation. Filters were extensively washed with PBST. The membrane was incubated with anti-Hsp20 (1:1000) for $1 \mathrm{~h}$ at room temperature in PBST $+1 \%$ skimmed dried milk powder. After six washes with PBST, the membrane was incubated with anti-rabbit secondary antibody alkaline phosphatase-conjugated (1:30000) (Sigma-Aldrich) and developed using NBT (Nitro Blue Tetrazolium)/BCIP (5bromo-4-chloroindol-3-yl phosphate) reagent (Promega).

\section{Acknowledgments}

We thank Dr Martin Haslbeck for helpful discussions. We also thank Dr M. Corvi, Dr G. Ward and Dr R. Dewey for critically reading this paper. This work was supported by: an ANPCyT (Agencia Nacional de Promoción Científica y Tecnológica) grant (BID1728 OC-ARPICT 05-34415; to S.O.A.), a Wood-Whelan Research Fellowships from the IUBMB (International Union of Biochemistry and Molecular Biology; to N.d.M.), travel allowances from Boehringer Ingelheim Fonds (to N.d.M.) and a CONICET (Consejo Nacional de Investigaciones Científicas y Técnicas)-NSF (National Science Foundation) collaborative grant. S.O.A. is a Researcher of the CONICET and of the Universidad 
Nacional de San Martin. N.d.M. is a Ph.D. Fellow of the CONICET. The contribution of Veronique Richard for electron microscopy is gratefully acknowledged.

\section{Abbreviations used}

$\begin{array}{ll}\text { DOC } & \text { deoxycholate } \\ \text { GAP } & \text { gliding-associated protein } \\ \text { GAP45 } & 45 \text { kDa GAP } \\ \text { HFF } & \text { human foreskin fibroblast } \\ \text { Hsp } & \text { heat-shock protein } \\ \text { HXGPRT } & \text { hypoxanthine-xanthine-guanine phosphoribosyl transferase } \\ \text { IFA } & \text { immunofluorescent assay } \\ \text { IMC } & \text { inner membrane complex } \\ \text { IMP } & \text { intramembranous particle } \\ \text { IP } & \text { immunoprecipitation } \\ \text { ORF } & \text { open reading frame } \\ \text { PFBS } & \text { fetal bovine serum in PBS } \\ \text { PPI } & \text { phosphatidylinositol phosphate } \\ \text { rHsp20 } & \text { recombinant Hsp20 } \\ \text { sHsp } & \text { small Hsp } \\ \text { TBS } & \text { Tris-buffered saline } \\ \text { TX-100 } & \text { Triton X-100 } \\ \text { YFP } & \text { yellow fluorescent protein. }\end{array}$

\section{References}

Arrigo AP. In search of the molecular mechanism by which small stress proteins counteract apoptosis during cellular differentiation. J. Cell. Biochem 2005;94:241-246. [PubMed: 15546148]

Achbarou A, Mercereau-Puijalon O, Autheman JM, Fortier B, Camus D, Dubremetz JF. Characterization of microneme proteins of Toxoplasma gondii. Mol. Biochem. Parasitol 1991;47:223-233. [PubMed: 1944419]

Bastin P, Bagherzadeh Z, Matthews KR, Gull K. A novel epitope tag system to study protein targeting and organelle biogenesis in Trypanosoma brucei. Mol. Biochem. Parasitol 1996;77:235-239. [PubMed: 8813669]

Cerede O, Dubremetz JF, Soete M, Deslee D, Vial H, Bout D, Lebrun M. Synergistic role of micronemal proteins in Toxoplasma gondii virulence. J. Exp. Med 2005;201:453-463. [PubMed: 15684324]

Cobb BA, Petrash JM. Structural and functional changes in the alpha A-crystallin R116C mutant in hereditary cataracts. Biochemistry 2000;39:15791-15798. [PubMed: 11123904]

Coppens I. Contribution of host lipids to Toxoplasma pathogenesis. Cell. Microbiol 2006;8:1-9. [PubMed: 16367861]

Coucheney F, Gal L, Beney L, Lherminier J, Gervais P, Guzzo J. A small HSP, Lo18, interacts with the cell membrane and modulates lipid physical state under heat shock conditions in a lactic acid bacterium. Biochim. Biophys. Acta 2005;1720:92-98. [PubMed: 16472556]

De Matteis MA, Godi A. PI-loting membrane traffic. Nat. Cell Biol 2004a;6:487-492. [PubMed: 15170460] 
De Matteis MA, Godi A. Protein-lipid interactions in membrane trafficking at the Golgi complex. Biochim. Biophys. Acta 2004b;1666:264-274. [PubMed: 15519320]

de Miguel N, Echeverria PC, Angel SO. Differential subcellular localization of members of the Toxoplasma gondii small heat shock protein family. Eukaryot. Cell 2005;4:1990-1997. [PubMed: 16339717]

Dobrowolski JM, Sibley LD. Toxoplasma invasion of mammalian cells is powered by the actin cytoskeleton of the parasite. Cell 1996;84:933-939. [PubMed: 8601316]

Dobrowolski JM, Niesman IR, Sibley LD. Actin in the parasite Toxoplasma gondii is encoded by a single copy gene, ACT1 and exists primarily in a globular form. Cell. Motil. Cytoskeleton 1997;37:253262. [PubMed: 9227855]

Foussard F, Leriche MA, Dubremetz JF. Characterization of the lipid content of Toxoplasma gondii rhoptries. Parasitology 1991;102:367-370. [PubMed: 1866182]

Garrido C, Gurbuxani S, Ravagnan L, Kroemer G. Heat shock proteins: endogenous modulators of apoptotic cell death. Biochem. Biophys. Res. Commun 2001;286:433-442. [PubMed: 11511077]

Gaskins E, Gilk S, DeVore N, Mann T, Ward G, Beckers C. Identification of the membrane receptor of a class XIV myosin in Toxoplasma gondii. J. Cell Biol 2004;165:383-393. [PubMed: 15123738]

Gilk SD, Raviv Y, Hu K, Murray JM, Beckers CJ, Ward GE. Identification of PhIL1, a novel cytoskeletal protein of the Toxoplasma gondii pellicle, through photosensitized labeling with 5-[ $\left.{ }^{125} \mathrm{I}\right]$ iodonaphthalene-1-azide. Eukaryot. Cell 2006;5:1622-1634. [PubMed: 17030994]

Gubbels MJ, Wieffer M, Striepen B. Fluorescent protein tagging in Toxoplasma gondii: identification of a novel inner membrane complex component conserved among Apicomplexa. Mol. Biochem. Parasitol 2004;137:99-110. [PubMed: 15279956]

Gupta N, Zahn MM, Coppens I, Joiner KA, Voelker DR. Selective disruption of phosphatidylcholine metabolism of the intracellular parasite Toxoplasma gondii arrests its growth. J. Biol. Chem 2005;280:16345-16353. [PubMed: 15708856]

Gusev NB, Bogatcheva NV, Marston SB. Structure and properties of small heat shock proteins (sHsp) and their interaction with cytoskeleton proteins. Biochemistry (Moscow) 2002;67:511-519. [PubMed: 12059769]

Hanada K, Kumagai K, Yasuda S, Miura Y, Kawano M, Fukasawa M, Nishijima M. Molecular machinery for non-vesicular trafficking of ceramide. Nature 2003;426:803-809. [PubMed: 14685229]

Haslbeck M, Franzmann T, Weinfurtner D, Buchner J. Some like it hot: the structure and function of small heat-shock proteins. Nat. Struct. Mol. Biol 2005;12:842-846. [PubMed: 16205709]

Hu K, Mann T, Striepen B, Beckers CJ, Roos DS, Murray JM. Daughter cell assembly in the protozoan parasite Toxoplasma gondii. Mol. Biol. Cell 2002a;13:593-606. [PubMed: 11854415]

$\mathrm{Hu}$ K, Roos DS, Murray JM. A novel polymer of tubulin forms the conoid of Toxoplasma gondii. J. Cell Biol 2002b;156:1039-1050. [PubMed: 11901169]

Johnson TM, Rajfur Z, Jacobson K, Beckers CJ. Immobilization of the typeXIV myosin complex in Toxoplasma gondii. Mol. Biol. Cell 2007;18:3039-3046. [PubMed: 17538016]

Mann T, Beckers C. Characterization of the subpellicular network, a filamentous membrane skeletal component in the parasite Toxoplasma gondii. Mol. Biochem. Parasitol 2001;115:257-268. [PubMed: 11420112]

Mann T, Gaskins E, Beckers C. Proteolytic processing of TgIMC1 during maturation of the membrane skeleton of Toxoplasma gondii. J. Biol. Chem 2002;277:41240-41246. [PubMed: 12177058]

Meissner M, Schluter D, Soldati D. Role of Toxoplasma gondii myosin A in powering parasite gliding and host cell invasion. Science 2002;298:837-840. [PubMed: 12399593]

Michell RH, Heath VL, Lemmon MA, Dove SK. Phosphatidylinositol 3,5-bisphosphate: metabolism and cellular functions. Trends Biochem. Sci 2006;31:52-63. [PubMed: 16364647]

Morrissette NS, Murray JM, Roos DS. Subpellicular microtubules associate with an intramembranous particle lattice in the protozoan parasite Toxoplasma gondii. J. Cell Sci 1997;110:35-42. [PubMed: 9010782]

Nakamoto H, Vigh L. The small heat shock proteins and their clients. Cell. Mol. Life Sci 2007;64:294306. [PubMed: 17187175] 
Parcellier A, Gurbuxani S, Schmitt E, Solary E, Garrido C. Heat shock proteins, cellular chaperones that modulate mitochondrial cell death pathways. Biochem. Biophys. Res. Commun 2003;304:505-512. [PubMed: 12729585]

Patron SA, Mondragon M, González S, Ambrosio JR, Guerrero BAL, Mondragon R. Identification and purification of actin from the subpellicular network of Toxoplasma gondii tachyzoites. Int. J. Parasitol 2005;35:883-894. [PubMed: 15970197]

Porchet E, Torpier G. Freeze fracture study of Toxoplasma and Sarcocystis infective stages. Z. Parasitenkd 1977;54:101-124. [PubMed: 415447]

Raiborg C, Rusten TE, Stenmark H. Protein sorting into multivesicular endosomes. Curr. Opin. Cell Biol 2003;15:446-455. [PubMed: 12892785]

Roth MG. Phosphoinositides in constitutive membrane traffic. Physiol. Rev 2004;84:699-730. [PubMed: 15269334]

Torok Z, Goloubinoff P, Horvath I, Tsvetkova NM, Glatz A, Balogh G, Varvasovszki V, Los DA, Vierling E, Crowe JH, Vigh L. Synechocystis HSP17 is an amphitropic protein that stabilizes heat-stressed membranes and binds denatured proteins for subsequent chaperone-mediated refolding. Proc. Natl. Acad. Sci. U.S.A 2001;98:3098-3103. [PubMed: 11248038]

Tsvetkova NM, Horvath I, Torok Z, Wolkers WF, Balogi Z, Shigapova N, Crowe LM, Tablin F, Vierling E, Crowe JH, Vigh L. Small heat-shock proteins regulate membrane lipid polymorphism. Proc. Natl. Acad. Sci. U.S.A 2002;99:13504-13509. [PubMed: 12368478]

van der ZE, Piekarski G. Endodyogeny in Toxoplasma gondii: a morphological analysis. Z. Parasitenkd 1967;29:15-35. [PubMed: 5601171]

Vivier E, Petitprez A. The outer membrane complex and its development at the time of the formation of daughter cells in Toxoplasma gondii. J. Cell Biol 1969;43:329-342. [PubMed: 5344151]

Yamashita S, Oku M, Wasada Y, Ano Y, Sakai Y. PI4P-signaling pathway for the synthesis of a nascent membrane structure in selective autophagy. J. Cell Biol 2006;173:709-717. [PubMed: 16754956] 


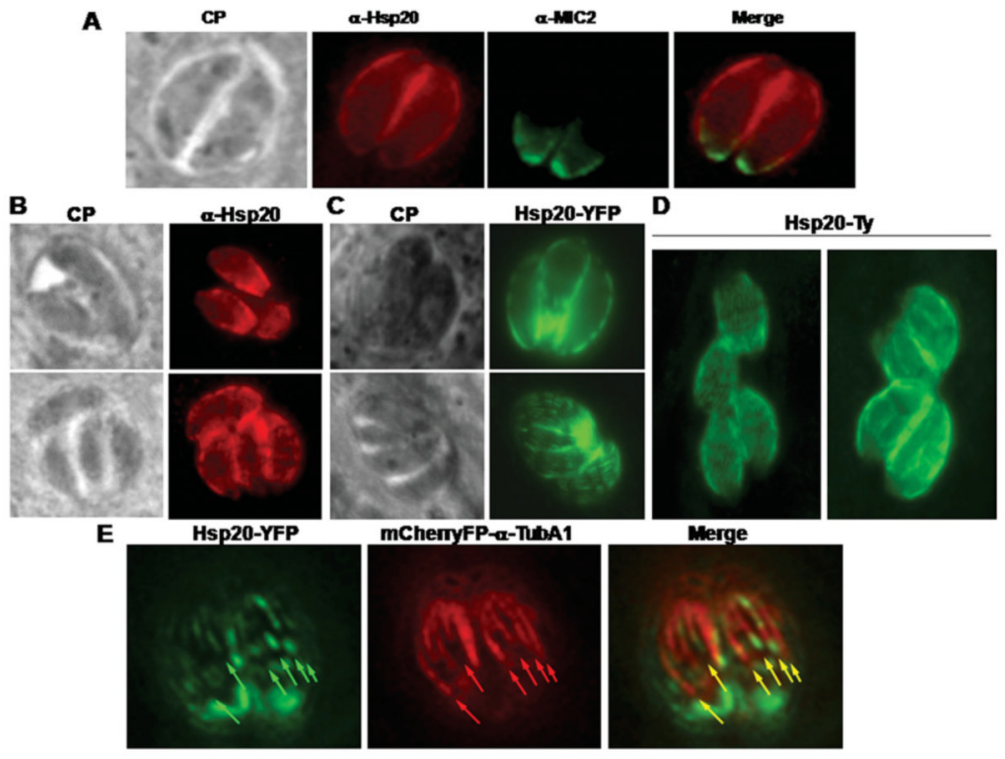

Figure 1. Hsp20 labelling patterns of wild-type and transgenic parasites

(A) Immunofluorescence analysis of intracellular parasites $24 \mathrm{~h}$ post-infection. The monolayer was permeabilized with $0.2 \% \mathrm{TX}-100$ and stained with rabbit anti-Hsp20 and monoclonal antiMIC2. Hsp20 antibody showed a similar pattern as IMC proteins with a weaker label in the MIC2 compartment. (B) Immunofluorescence analysis with anti-Hsp20 also labelled developing daughter cells (upper panel) and an optical section facing the parasite surface shows the discontinuous stripes along the body of the parasite. (C) Living Hsp20-YFP also gave a pattern associated with the IMC. In the lower panel, an optical section facing the parasite surface shows that Hsp20-YFP is forming discontinuous stripes down the body of the parasite. (D) Intracellular Hsp20-Ty-transfected tachyzoites were incubated with the monoclonal antibody anti-Ty. The left panel shows the discontinuous stripes along the body of the parasite. In the right panel, Hsp20 is detected in daughter cells of replicating parasites. (E) Colocalization of parasites transfected with Hsp20-YFP and mCherryFP- $\alpha$-TUBA1. Hsp20 stripes follow the same trajectories as the cortical microtubules (arrows). 


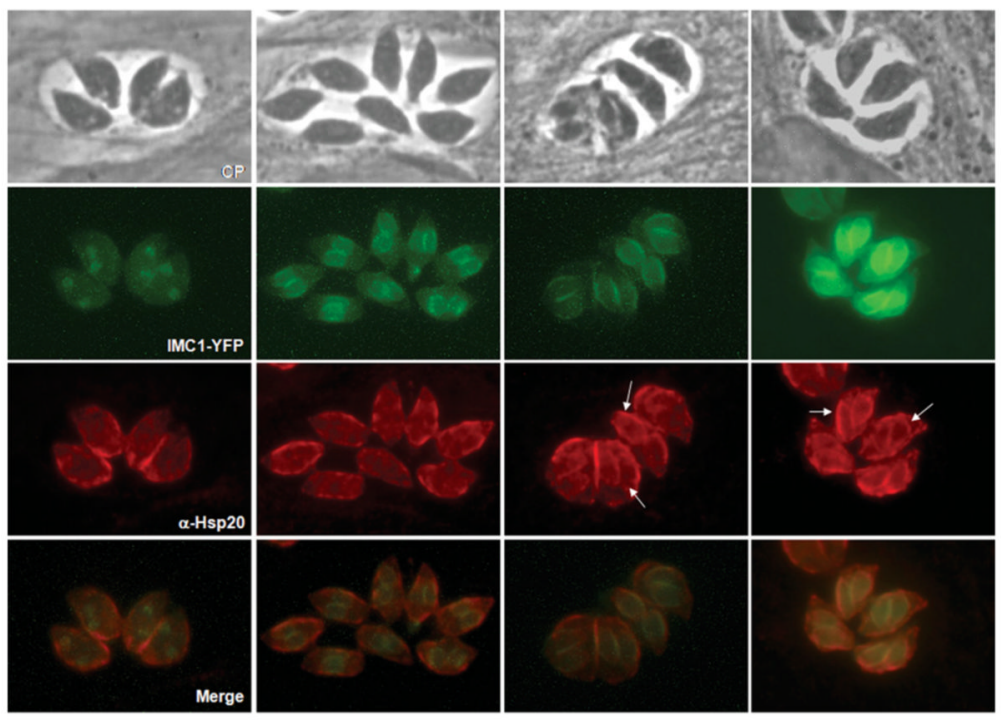

Figure 2. Examination of replicating parasites with anti-Hsp20 antibody

IMC1 was used to visualize the network in all stages of parasite cell division. The network of the new daughter cells can be detected at a very early stage of endodyogeny with IMC1-YFP parasites (IMC1 panel). Hsp20 reveals labelling of the mother cell IMC until the time of budding, and its labelling is also apparent in the daughters at late stages of endodyogeny (white arrows). 


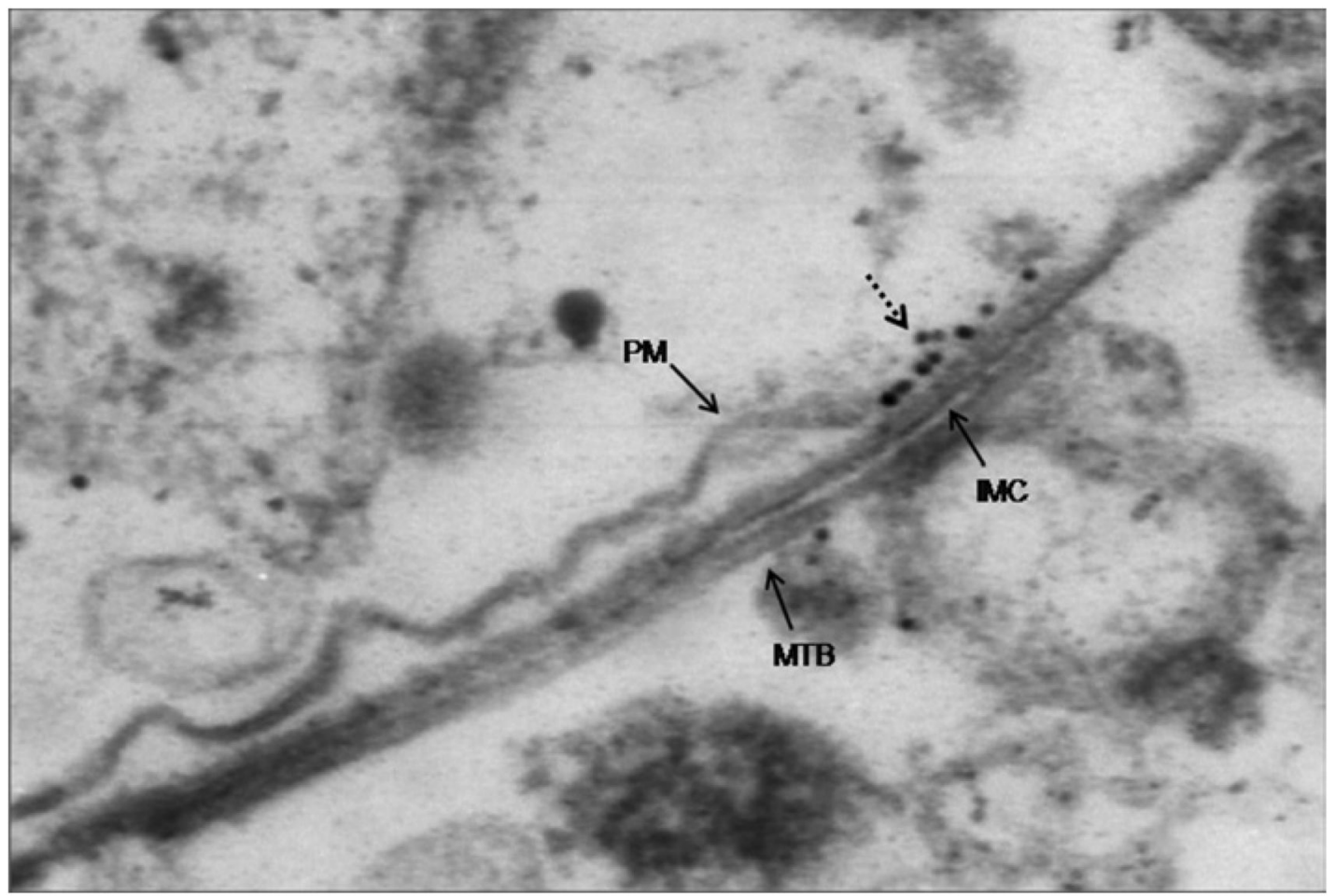

Figure 3. Ultrastructural localization of Hsp20

Isolated pellicle preparations obtained by parasite sonication were incubated with anti-Hsp20 serum, followed by goat anti-rabbit secondary antibodies conjugated with 10 -nm gold. The Hsp20 label is located in the IMC facing the plasma membrane. The arrows indicate the plasma membrane (PM), the IMC membranes (IMC) and the microtubules (MTB). Dot arrows indicate Hsp20 localization. 

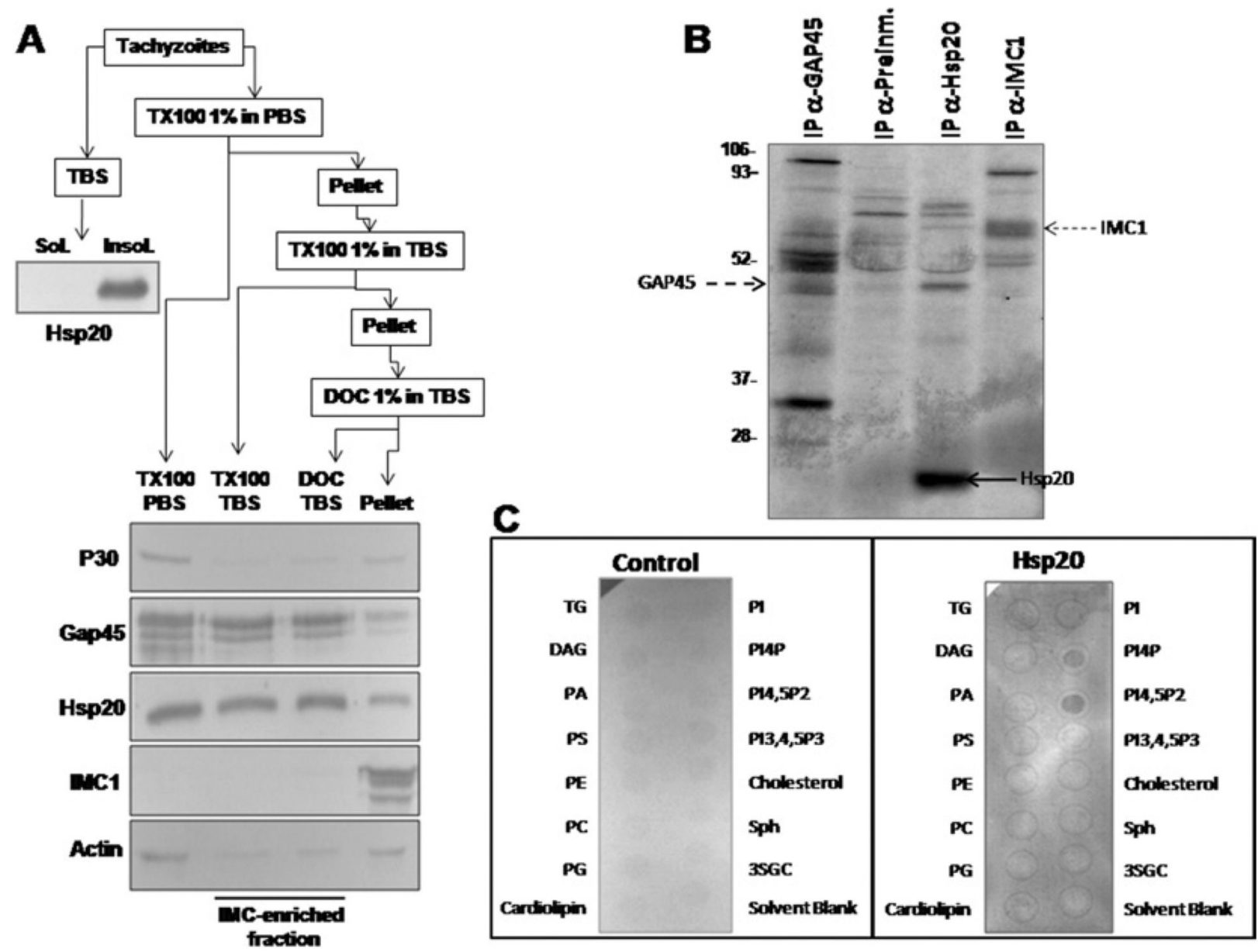

Figure 4. Biochemical analysis of $T$. gondii Hsp20

(A) Detergent extraction profile of Hsp20. Parasites were sequentially extracted with $1 \%$ TX-100 and 1\% DOC, and analysed by Western blotting using antibodies against Hsp20, IMC1, SAG1 (P30), actin and GAP45. The extraction profiles of Hsp20 are very similar to those of GAP45; both are found in the IMC-enriched fraction. (B) Analysis of proteins interacting with Hsp20. IP analysis with the anti-Hsp20 serum. Intracellular parasites were metabolically labelled with $\left[{ }^{35} \mathrm{~S}\right]$ methionine and $\left[{ }^{35} \mathrm{~S}\right]$ cysteine, lysed in a buffer containing TX-100, and subjected to IP using either anti-Hsp20 serum or pre-immune serum (as a negative control). As Hsp20 is located in the IMC, IP assays using anti-GAP45 and anti-IMC1 antibodies were also performed. Anti-Hsp20 antibody immunoprecipitated mostly Hsp20 protein. AntiGAP45 and -IMC1 antibodies did not pull down any protein in the region of Hsp20. (C)

Analysis of lipid interaction of Hsp20 by lipid-membrane overlay assays. Phospholipid strips were probed with $10 \mu \mathrm{g} / \mathrm{ml}$ of Hsp20 recombinant protein. Control experiments were performed following the same procedure except that Hsp20 was omitted. Abbreviations: PA, phosphatidic acid; PG, phosphatidylglycerol; Sph, sphingomyelin; PI, phosphatidylinositol; PI4P, phosphatidylinositol 4-phosphate; PI4,5P2, phosphatidylinositol 4,5-bisphosphate; PI3,4,5P2, phosphatidylinositol 3,4,5-trisphosphate; PS, phosphatidylserine; DAG, diacylglycerol; TG, triacylglycerol; PE, phosphatidylethanolamine; PC, phosphatidylcholine; 3SGC, 3-sulfogalactosylceramide. In all cases, the results shown are representative of at least three independent experiments. 